\title{
Identificação e cálculos de parâmetros de soldagem aplicados a pontes ferroviárias
}

\author{
Identification and calculations of welding parameters applied to railway bridges \\ Identificación y cálculo de parámetros de soldadura aplicados a puentes ferroviarios
}

Recebido: 06/04/2021 | Revisado: 14/04/2021 | Aceito: 19/04/2021 | Publicado: 04/05/2021

Camila Ferreira da Silva
ORCID: https://orcid.org/0000-0002-6141-9556
Universidade Federal Fluminense, Brasil
E-mail: camilaferreira@id.uff.br
Ronaldo Gomes de Castro Medeiros
ORCID: https://orcid.org/0000-0002-7988-8289
E-mail: ronaldogcm@yahoo.com.br
Pontifícia Universidade Católica do Rio de Janeiro, Brasil
Patrícia Guimarães Monteiro de Freitas
ORCID: https://orcid.org/0000-0001-5946-8984
Pontifícia Universidade Católica do Rio de Janeiro, Brasil
E-mail: patricia-gmf@hotmail.com
Nathália de Paula Sousa
ORCID: https://orcid.org/0000-0001-9260-3762
Universidade Federal Fluminense, Brasil
E-mail: nathalia_sousa@id.uff.br
Isadora Rapoza Zenóbio
ORCID: https://orcid.org/0000-0001-5068-4701
Universidade Federal Fluminense, Brasil
E-mail: isadora_zenobio@hotmail.com

\section{Resumo}

Pontes ferroviárias são elementos chave no cenário do transporte mundial, apresentando uma variedade de aplicações possíveis. Entretanto, faz-se necessário averiguar o desempenho da sua estrutura a fim de prevenir danos em termos de projeto, pois caso o projeto seja aplica sem que haja uma verificação de sua estrutura e seus componentes pode-se ocorrer acidentes indesejáveis. Os parâmetros essenciais a serem investigados são o teor de carbono equivalente do aço (CEq), a Temperatura de Pré Aquecimento (TPA), a Energia Liquida de Soldagem (Hl) e o processo de soldagem em si, uma vez que os mesmos influenciam nas propriedades mecânicas do material. Este artigo aborda a determinação dos parâmetros de soldagem para uma ponte ferroviária, sendo estes obtidos a partir da composição química do aço estrutural da ponte. Determina-se ainda questões de dimensionamento, tonelagem e a escolha do processo de soldagem ideal para um projeto de grande magnitude, exemplificando procedimentos técnicos inerentes à construção.

Palavras-chave: Parâmetros de soldagem; Carbono equivalente; Eletrodo revestido.

\begin{abstract}
Railway bridges are key elements in the world transport landscape, presenting a variety of possible applications. However, it is necessary to check the performance of its structure in order to prevent damage in terms of design, because if the project is applied without there being a verification of its structure and its components, undesirable accidents may occur. The essential parameters to be investigated are the equivalent carbon content of the steel (CEq), the Preheating Temperature (TPA), the Net Welding Energy (Hl) and the welding process itself, since they influence the mechanical properties of the material. This the article addresses the determination of welding parameters for a railway bridge, which are obtained from the chemical composition of the structural steel of the bridge. It also determines issues of dimensioning, tonnage and the choice of the ideal welding process for a project of great magnitude, exemplifying technical procedures inherent to the construction.
\end{abstract}

Keywords: Welding parameters; Equivalent carbon content; Shielded metal arc welding.

\section{Resumen}

Los puentes ferroviarios son elementos clave en el panorama mundial del transporte y presentan una variedad de posibles aplicaciones. Sin embargo, es necesario verificar el desempeño de su estructura para evitar daños en términos de diseño, ya que si el proyecto se aplica sin que exista una verificación de su estructura y sus componentes, pueden ocurrir accidentes indeseables. Los parámetros esenciales a investigar son el contenido de carbono equivalente del acero (CEq), la Temperatura de Precalentamiento (TPA), la Energía Neta de Soldadura (Hl) y el propio proceso de soldadura, ya que influyen en las propiedades mecánicas del material. Este artículo aborda la determinación de los parámetros de 
soldadura para un puente ferroviario, que se obtienen a partir de la composición química del acero estructural del puente. También determina cuestiones de dimensionamiento, tonelaje y la elección del proceso de soldadura ideal para un proyecto de gran envergadura, ejemplificando procedimientos técnicos inherentes a la construcción.

Palabras clave: Parámetros de soldadura; Equivalente de carbono; Electrodo revestido.

\section{Introdução}

As pontes ferroviárias possuem uma grande importância para o transporte mundial devido a sua vasta aplicação, entretanto, sua construção não é tão simples. Um dos principais fatores para seu desenvolvimento é a soldagem de suas juntas, que se configura como um processo complexo devido ao perfil dos trilhos e o tamanho de sua seção transversal (Marsh, 2021) (Barbosa, 2009).

Referente a soldagem, deve-se levar em consideração diversos parâmetros de soldagem, tais quais o teor de carbono equivalente do aço (CEq), a Temperatura de Pré Aquecimento (TPA), a Energia Liquida de Soldagem (HI) e fatores estruturais (Keehan et al., 2006) (Atinks et al., 2002).

A determinação destes parâmetros é de suma importância em um projeto de soldagem estrutural uma vez devido a união metálica em altas temperaturas podem ocorrer modificações microestruturais no aço, impactando em suas propriedades mecânicas como a fissuração a frio e resistência ao desgaste (Fujii, 2015). Neste contexto os elementos de liga representam papel importante, pois está associado a sensibilidade a fragilização por hidrogênio e a TPA (Góis, 2002) (Oliveira, 2001) (Carlos et al., 2007) (Melo et al., 2020) (Melo et al., 2020).

Por motivos microestruturais quanto maior o CEq de um aço, maior a tendência à fissuração a frio. $\mathrm{O}$ teor de carbono prejudica a soldabilidade fragilizando a região da junta e diminuindo a tenacidade da solda, de modo que a utilização de uma TPA adequada e/ou o aumento da $\mathrm{Hl}$ são determinantes para evitar a ocorrência de microestrutura susceptível. Ainda, ao determinar CEq pode-se prever o comportamento do aço quando submetido à um ciclo térmico e adotar um processo de soldagem que não irá comprometer a qualidade da solda (Anderson, 2005) (Gomes et al., 2020).

O presente artigo aborda o projeto e identificação de parâmetros de soldagem visando seu emprego em uma ponte ferroviária, de modo a propor uma aplicação que minimize os fenômenos danosos sua estrutura metálica. Considera-se para o ambiente de construção dois tipos de união das juntas, a soldagem por arco submerso (SAW) e por eletrodo revestido (ER).

No processo SAW o calor requerido para fundir o metal é gerado por um arco formado pela corrente elétrica passando entre o arame de soldagem e a peça de trabalho. Um dos pontos importantes relacionados ao arco elétrico nesta técnica é que não há arco visível, fumos, respingos ou faíscas (Wainer, 1992). Assim, as perdas do consumível (arame de soldagem) são desprezíveis.

A soldagem por ER figura como o mais empregado dos processos de soldagem, onde o calor gerado pelo arco elétrico é mantido na extremidade do eletrodo metálico revestido e da peça a ser trabalhada, sendo o responsável por fundir a alma do eletrodo, o metal de base e o revestimento (Modenesi, 2008) (Augusto et al., 2017).

Este artigo surge com o objetivo de determinar com segurança a energia líquida de soldagem recomendada, assim como a faixa de uso de pré-aquecimento no material com base nas especificações de composição química do aço estrutural. Ainda, realizar o planejamento da tonelagem necessária para a estrutura e do procedimento de soldagem considerando ER e SAW.

\section{Metodologia}

O presente estudo refere-se a uma pesquisa com abordagem qualitativa, com objetivo de pesquisa descritivo, visando determinar os parâmetros de soldagem obtidos a partir da composição química de um aço estrutural para aplicações em pontes ferroviárias. (Soares et al., 2018) (Carlos, 2011). 


\subsection{Especificação das chapas metálicas}

Considera-se o ASTM A242, aço estrutural patinável de alta resistência e baixa liga (ARBL) (Aços Patináveis, 2021) (Revie, 2011). Este material apresenta um limite de escoamento mínimo de 345 MPa e limite de resistência mínimo de 480 MPa, sendo largamente utilizado na construção de pontes e viadutos do mundo inteiro (CBCA, 2020). A Tabela 1 exibe sua composição química.

Tabela 1 - Composição química do aço ASTM A242.

\begin{tabular}{cccccc}
\hline Composição Química & $\mathbf{C}$ & Mn & $\mathbf{P}$ & $\mathbf{S}$ & $\mathbf{C u}$ \\
\hline$(\mathrm{w} \%)$ & 0,15 & 1,00 & 0,15 & 0,05 & 0,20 \\
\hline
\end{tabular}

Fonte: Autores.

\subsection{Especificações Estruturais}

Neste estudo, considera-se necessária a confecção de 10 módulos de 4 vigas cada visando a montagem da ponte. A tabela 2 mostra detalhadamente as dimensões de cada chapa, no intuito de produzir uma viga com alma (chapa lateral) e flanges (chapas superiores e inferiores) nas dimensões industriais especificadas. Considera ainda as dimensões dos enrijecedores de alma necessários as vigas em I.

Tabela 2 - Dimensões para a montagem de um módulo individual de viga em I.

\begin{tabular}{ccccc}
\hline \multicolumn{5}{c}{ Montagem de um Módulo Estrutural } \\
\hline Peça & Espessura (m) & Largura (m) & Comprimento (m) & Quantidade por módulo \\
\hline Chapa X (superior) & 0,03 & 3,5 & 7 & 1 \\
\hline Chapa Y (lateral) & 0,02 & 7 & 7 & 1 \\
\hline Chapa Z (inferior) & 0,03 & 3,5 & 7 & 8 \\
\hline Enrijecedor & 0,012 & 0,02 & 7 & 1 \\
\hline
\end{tabular}

Fonte: Autores.

Faz- se necessário considerar o uso de enrijecedores nas chapas para fins de sustentação. O cálculo da tonelagem necessária (Equação 1) ao projeto considera 80 chapas para a produção dos flanges, 40 chapas para a produção das almas e aço adicional relacionado a produção de reforços.

$$
\mathrm{P}=\mathrm{V} \times \mathrm{Q} \times \mathrm{D}
$$

Onde $\mathrm{P}$ representa o peso (ton), V representa o volume da chapa $\left(\mathrm{m}^{3}\right)$, Q representa a quantidade por módulo, D representa a densidade do material empregado e ainda, a densidade do aço por tabela $=7,87 \mathrm{t} / \mathrm{m}^{3}$.

\subsection{Processos de Soldagem}

Os processos de soldagem considerados são eletrodo revestido e arco submerso, cujas especificações de funcionamento dos equipamentos empregados na soldagem são exibidas na Tabela 3. 
Tabela 3 - Parâmetros dos processos de soldagem.

\begin{tabular}{cccc}
\hline Grandeza & Eletrodo Revestido & Arco Submerso & Unidade \\
\hline Tensão $(\mathrm{V})$ & 14 & 20 & $\mathrm{~V}$ \\
\hline Corrente $(\mathrm{I})$ & 160 & 500 & $\mathrm{~A}$ \\
\hline Velocidade $(v)$ & 15 & 70 & $\mathrm{~cm} / \mathrm{min}$ \\
\hline
\end{tabular}

Fonte: Autores.

\subsection{Determinação do Tipo de Chanfro e Tipo de Junta Soldada}

As juntas adequadas para a confecção da estrutura soldada são de topo e de filete. Quanto aos chanfros, recomenda-se chanfros em $\mathrm{V}$ nas soldas $\mathrm{A}, \mathrm{B}$ e $\mathrm{C}$ e chanfro reto nas juntas $\mathrm{D}$ e $\mathrm{E}$.

Figura 1 - Ilustração da geometria dos chanfros e posições.
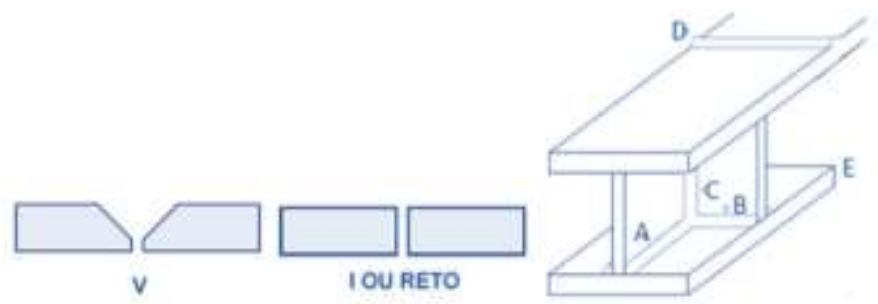

Fonte: Autores.

\subsection{Cálculo do Carbono Equivalente (CEq)}

Os 2 principais métodos de cálculo de carbono equivalente são o Método de Yurioka (Equação 2) e o método Pcm (Equação 3):

$$
\begin{gathered}
\mathrm{CEN}=\% \mathrm{C}+\mathrm{A}(\mathrm{C}) *\left[\frac{\% \mathrm{Si}}{24}+\frac{\% \mathrm{Mn}}{6}+\frac{\% \mathrm{Cu}}{15}+\frac{\% \mathrm{Ni}}{20}+\left(\frac{\% \mathrm{Cr}+\% \mathrm{Mo}+\% \mathrm{Nb}+\% \mathrm{~V}}{5}\right)+5 \% \mathrm{~B}\right] \\
\mathrm{A}(\mathrm{C})=0,75+0,25 * \operatorname{tgh}[20(\mathrm{C}-0,12)]
\end{gathered}
$$

Onde $\mathrm{A}(\mathrm{C})$ é o fator de correção associado ao cálculo.

$$
\mathrm{Pcm}=\% \mathrm{C}+\frac{\% \mathrm{Si}}{30}+\left(\frac{\% \mathrm{Mn}+\% \mathrm{Cu}+\% \mathrm{Cr}}{20}\right)+\frac{\% \mathrm{Ni}}{60}+\frac{\% \mathrm{Mo}}{15}+\frac{\% \mathrm{~V}}{10}+5 \% \mathrm{~B}
$$

A equação (4) de Pcm é a mais adequada para aços com menor teor de carbono $(\mathrm{C} \leq 0.16 \%)$. Já a fórmula CEN é adequada para aços com teores de carbono entre 0,01 a 0,30\% (Yurioka, 2001). O fator de correção A(C) permite que CEN seja aplicada a ambos os tipos de aço.

\subsection{Cálculo do Calor Adicionado (CA) da Energia Líquida de Soldagem (HI)}

$H_{l}$ é calculada numa relação inversa com CA, onde quanto maior o calor adicionado (CA), menor deve ser temperatura de pré-aquecimento (TPA) empregada na solda. 


$$
\mathrm{Hl}=\mathrm{n} \frac{\mathrm{VI}}{\mathrm{v}}
$$

Onde I é a corrente aplicada em ampères, $\mathrm{V}$ a tensão em volts e $v$ a velocidade empregada em $\mathrm{cm} / \mathrm{min}$ no cordão de solda. Para fins de cálculo, cabe salientar que $1 \mathrm{VA}$ equivale a $1 \mathrm{~J} / \mathrm{s}$. $\eta$ é o rendimento do processo de soldadura a considerar. A Tabela 4 indica os valores típicos para $\eta$.

Tabela 4 - Rendimento dos processos de soldagem.

\begin{tabular}{cccccc}
\hline \multicolumn{5}{c}{$\eta$ do Processo } \\
\hline Eletrodo Revestido & GMAW & GTAW (CC+) & GTAW (CA) & SAW & Oxi-gás \\
\hline $0,65-0,85$ & $0,65-0,85$ & $0,50-0,80$ & $0,20-0,50$ & $0,80-0,99$ & $0,25-0,80$ \\
\hline
\end{tabular}

Fonte: Autores.

No presente estudo consideramos os rendimentos térmicos máximos alcançados pelos processos em questão, sendo $\eta$ de $85 \%$ para eletrodo revestido e $99 \%$ para arco submerso (SAW).

\subsection{Cálculo da Temperatura de Pré Aquecimento (TPA)}

O comportamento da soldagem depende do comportamento de alguns parâmetros, sendo um deles a temperatura de préaquecimento (Delgado, 2014).

$$
\operatorname{TPA}(\stackrel{\mathrm{o}}{\mathrm{C}})=350 * \sqrt{(\mathrm{Ceq}}-0,25)
$$

\subsection{Procedimento de Soldagem dos perfis estruturais}

A primeira etapa consiste em soldar os flanges e almas conforme a Figura 2-a através de soldagem à arco submerso mecanizado, fazendo uso de trator de soldagem e/ou o auxílio de uma ponte rolante para o posicionamento adequado da alma no flange conforme a Figura 2-b. A ponte rolante pode manusear a placa com uma espécie de pinça, que mantém a integridade da chapa; ou com cabos, sendo necessário fazer furos na chapa.

Para a realização da soldagem do flange na outra extremidade da alma faz-se necessária uma ponte rolante com garras, promovendo a rotação de $180^{\circ}$ do perfil em T obtido com a primeira solda de filete.

Figura 2 - (a) Primeira etapa de soldagem; (b) Segunda etapa de soldagem.

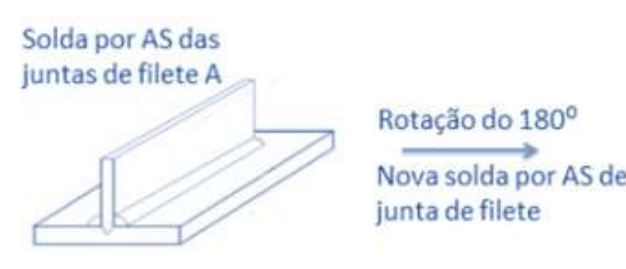

(a)

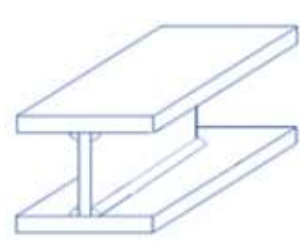

(b)

Fonte: Autores.

A realização da solda B requer um carro com garras guia para o transporte da chapa e posicionamento no chanfro. Assim, pode-se proceder a soldagem B (horizontal) conforme a Figura 3-a, e posteriormente a soldagem C (vertical) conforme a Figura 3-b, desta vez através de eletrodos revestidos. 
Figura 3 - (a) Esquema de soldagem em filete da junta B; (b) Esquema de soldagem em filete da junta C.

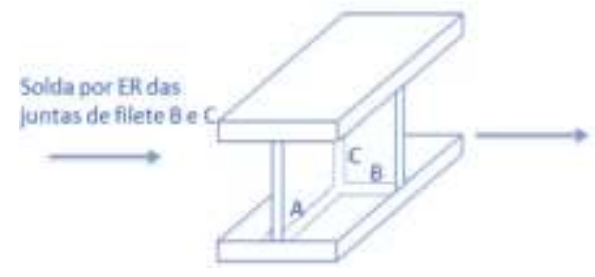

(a)

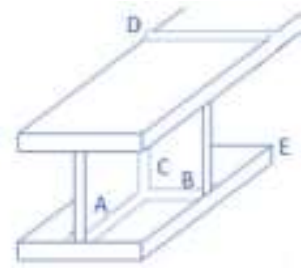

(b)

Fonte: Autores.

A solda de topo D deve ser realizada em campo utilizando o processo de arco submerso. Para realizar essa solda, o mais apropriado é a utilização de um trator de soldagem devido a planicidade das chapas e simplicidade de soldagem, o que irá garantir uma qualidade do cordão de solda satisfatória, além de garantir maior velocidade de produção.

\section{Resultados e Discussão}

\subsection{Especificações Estruturais}

A Tabela 5 exibe as tonelagens de aço requeridas para a confecção de um módulo e o total para as estruturas soldadas da ponte ferroviária, obtidas por meio da Equação 1.

Tabela 5 - Tonelagem de aço necessária à construção das estruturas soldadas.

\begin{tabular}{ccc}
\hline & Peso (ton) \\
\hline Peça & 1 Módulo & 10 Módulos \\
\hline Chapa X & 5,784 & 57,845 \\
\hline Chapa Y & 7,713 & 77,126 \\
\hline Chapa Z & 5,784 & 57,845 \\
\hline Enrijecedor & 0,106 & 1,058 \\
\hline Peso Total & 19,387 & 193,873 \\
\hline
\end{tabular}

Fonte: Autores.

\subsection{Cálculo da Temperatura de Pré Aquecimento e Carbono Equivalente (CEq)}

A Tabela 6 evidencia os valores encontrados para o cálculo do carbono equivalente pelos dois métodos. O fator de correção AC a ser considerado para o método CEN é de 0,884 .

Tabela 6 - Composição química do aço ASTM A242.

\section{Método Carbono Equivalente TPA}

\begin{tabular}{ccc}
\hline EN & 0,31 & 85,14 \\
\hline PCM & 0,21 & 70,00
\end{tabular}

Fonte: Autores. 
Conforme especificações de composição química do aço, ambos os métodos são aplicáveis. Entretanto, opta-se pela adoção do método PCM para fins de baixo consumo de energia, otimizando a redução da TPA.

\subsection{Energia Líquida de Soldagem (HI)}

O processo de arco submerso obteve uma energia líquida de soldagem maior do que o processo de eletrodo revestido, devido ao seu rendimento.

Tabela 7 - Calor Adicionado ou Energia Líquida de Soldagem.

\begin{tabular}{cc}
\hline \multicolumn{3}{c}{ Hl $(\mathbf{k J} / \mathbf{m m})$} \\
\hline AS & 0,849 \\
\hline ER & 0,762 \\
\hline \multicolumn{2}{c}{ Fonte: } \\
Autores.
\end{tabular}

\section{Considerações Finais}

O projeto de soldagem de estruturas metálicas envolve uma série de parâmetros relevantes a níveis de metalurgia da soldagem. Estes parâmetros são severamente influenciados pela especificação do aço estrutural, envolvendo o tipo de solda mais adequado a aplicação. No que tange o projeto de uma estrutura metálica ferroviária, a soldagem por arco submerso e eletrodo revestido mostram-se mais adequadas considerando suas baixas perdas e alto rendimento em campo quando comparados a outros processos de soldagem (ER 85\% e SAW 99\%).

Outro ponto importante a ser considerado é determinação da tonelagem das chapas utilizadas na estrutura, obtido através do dimensionamento do peso total da estrutura, incluindo a sustentação das vigas em I por enrijecedores.

Um parâmetro de soldagem de grande relevância é o cálculo do carbono equivalente. Com a adoção do método PCM, vinculada a composição química do aço, foi possível dimensionar uma temperatura de pré aquecimento TPA de $70^{\circ} \mathrm{C}$ com uma economia de aporte térmico em torno de $15^{\circ} \mathrm{C}$.

Foi possível identificar e indicar vários parâmetros de soldagem como requisitos para projetos estruturais de vias de transporte, considerando a obtenção de estruturas soldadas com a qualidade metalúrgica adequada a uma ponte ferroviária.

Por fim, sugere-se como um possível tema para trabalho futuro a modificação do aço utilizado e a análise de quais mudanças ocorreriam nos parâmetros de soldagem, pois como foi visto ao longo do artigo a quantidade de carbono terá influência direta na obtenção desses parâmetros. Similarmente interessante seria a avaliação dos efeitos térmicos na microestrutura do aço estrutural, assim como a dissipação de tensões residuais quando do emprego dos processos de soldagem com os parâmetros aqui recomendados.

\section{Referências}

Aços Estruturais | Centro Brasileiro da Construção em Aço. CBCA. (2020). Retrieved 15 April 2021, from https://www.cbca-acobrasil.org.br/site/acosestruturais/.

Aços Patináveis (Corten) | Aços Patináveis | Aços e Ferros Fundidos | Aços \& Ligas | Infomet. Infomet. (2021).

Anderson, T. (2005). Fracture Mechanics (3rd ed.). Chapman and Hall/CRC.

Atkins, G., Thiessen, D., Nissley, N., \& Adony, Y. (2002). Welding Process Effects in Weldability Testing of Steels. Welding Research, 61-68.

Augusto Rocha, A., Wilson Gomes Pimenta, L., Magalhães Rocha, G., \& Gomes de Cerqueira, T. (2017). Análise de Soldagem por Eletrodo Revestido do Tipo Rutílico, Básico e Celulósico. Revista Científica Multidisciplinar Núcleo Do Conhecimento, 1(2), 684-690.

Barbosa, R. (2009). Processos de Fabricação: Soldagem e Fundição. Centro Universitário do Leste de Minas de Gerais. Coronel Fabriciano - MG. 
Research, Society and Development, v. 10, n. 5, e22310514727, 2021

(CC BY 4.0) | ISSN 2525-3409 | DOI: http://dx.doi.org/10.33448/rsd-v10i5.14727

Carlos F. Jorge, J., G. de Souza, L., Santos Filho, O., Santos Filho, A., \& S. Bott, I. (2007). Influência da composição química e tratamento térmico póssoldagem nas propriedades mecânicas e microestruturais de metais de solda de alta resistência. In XXXIII Congresso Nacional de Soldagem.

Carlos Köche, J. (2011). Fundamentos de metodologia científica: teoria da ciência e iniciação à pesquisa. Editora Vozes.

Delgado Júnior, H., Alvarenga Palmeira, A., Fernandes Habibe, A., \& Oliveira Magnago, R. (2014). Estudo experimental das deformações residuais oriundas do processo de soldagem. Cadernos Unifoa, Edição Especial do Curso de Mestrado Profissional em Materiais.

Fujii, M., Nakanowatari, H., \& Nariai, K. (2015). Rail Flash-Butt Welding Technology. JFE Technical Report, $159-163$.

Góis Luciano de Azevedo, A. (2002). Aplicação da técnica da dupla camada na soldagem do aço ABNT 1045 (Master). Universidade Federal do Ceará.

Gomes, A., Jorge, J., Souza, L., Bott, I., Araújo, L., \& Mendes, M. (2020). Desempenho de metal de solda adequado para soldagem de componentes de ancoragem. Matéria (Rio De Janeiro), 25(2). https://doi.org/10.1590/s1517-707620200002.1046

H. Marsh. J. Railway History | The Canadian Encyclopedia. Thecanadianencyclopedia.ca. Retrieved 14 April 2021, from https://www.thecanadianencyclopedia.ca/en/timeline/railway-history.

J. Modenesi, P. (2008). Soldabilidade de Algumas Ligas Metálicas [Ebook]. Universidade Federal de Minas Gerais.

Keehan, E., Karlsson, L., Andrén, H., \& Bhadeshia, H. (2006). Influence of carbon, manganese and nickel on microstructure and properties of strong steel weld metals: Part 3 - Increased strength resulting from carbon additions. Science And Technology Of Welding And Joining, 11(1), 19-24. https://doi.org/10.1179/174329306x77858

Melo, R., Maia, A., Rocha Junior, D., Carvalho, A., Silva, F., Queiroz, P., \& Souza, I. (2020). Obtenção numérica do perfil térmico durante passagem de fonte de calor nos materiais AISI 410, 304L e 430. Research, Society And Development, 9(7), e63973884. https://doi.org/10.33448/rsd-v9i7.3884

Melo, R., Souza, I., Maia, A., Silva, F., Queiroz, P., \& Rocha Junior, D. et al. (2020). Aquisição numérica do perfil tensão deformação durante passagem de fonte de calor nos materiais AISI 410, 304L e 430. Research, Society And Development, 9(7), e286974224. https://doi.org/10.33448/rsd-v9i7.4224

Oliveira, S., \& Miranda, P. (2001). Concentração crítica de hidrogênio para a fragilização pelo hidrogênio em aços. Matéria (Rio De Janeiro), (5).

Revie, R., \& Uhlig, H. (2011). Uhlig's corrosion handbook. Wiley.

Soares Pereira, A., Moreira Shitsuka, D., José Parreira, F., \& Shitsuka, R. (2018). Metodologia da pesquisa científica. Universidade Federal De Santa Maria.

Wainer, E., Brandi, S., \& Mello, F. (1992). Soldagem: Processos e Metalurgia. Edgard Blücher.

Yurioka, N. (2001). Advances in Physical Metallurgy and Processing of Steels. Physical Metallurgy of Steel Weldability. ISIJ International, 41(6), 566-570. https://doi.org/10.2355/isijinternational.41.566 